\title{
Diverticulosis colónica
}

\section{Colonic diverticulum}

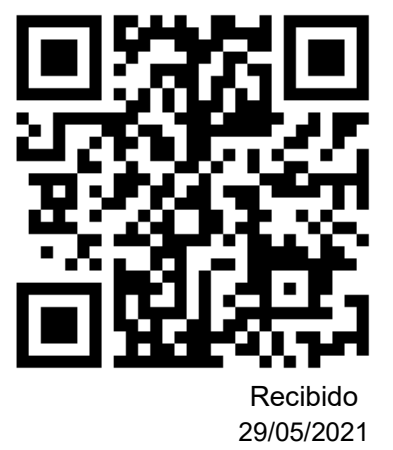

${ }^{1}$ Dra. Priscilla Montoya Alan Investigadora independiente, San José, Costa Rica

(iD https://orcid.org/0000-0002-0216-8961

${ }^{2}$ Dra. Katherine Murillo Alvarado Investigadora independiente, San José, Costa Rica

(I) https://orcid.org/0000-0002-7156-4554

Corregido $10 / 06 / 2021$
Aceptado 20/06/2021

\section{RESUMEN}

La diverticulosis cólica se define por una anomalía anatómica adquirida del colon, que se caracteriza por la presencia de seudodivertículos formados por herniaciones de la mucosa y submucosa a través de la capa muscular del colon.

Es una de las patologías más comunes a nivel del intestino grueso en el mundo occidental y su prevalencia durante el último siglo se ha incrementado probablemente debido a cambios en los estilos de vida.

La enfermedad diverticular del colon es una condición común con presentación clínica variable y su presentación clínica abarca desde ser asintomática hasta la presencia de síntomas y el desarrollo de diversas complicaciones.

Generalmente el diagnóstico se realiza de forma incidental, al realizar una colonoscopia o enema con medio de contraste.

Los casos asintomáticos no requieren tratamiento y los pacientes sintomáticos se realiza modificaciones en la dieta y tratamiento oral. Las complicaciones requieren un manejo específico, ya sea con tratamiento antibiótico o quirúrgico.

PALABRAS CLAVE: diverticulosis; mucosa; intestino grueso; colonoscopia; antibiótico.

\section{ABSTRACT}

Colic diverticulum is defined by an acquired anatomical abnormality of the colon, characterized by the presence of pseudodiverticula formed by herniations of the mucosa and submucosa through the muscular layer of the colon.

It is one of the most common pathologies of the large intestine in the Western world and its prevalence during the last century has probably increased due to changes in lifestyle. 
Diverticular disease of the colon is a common condition with variable clinical presentation and its clinical presentation ranges from being asymptomatic to the presence of symptoms and the development of various complications.

Usually the diagnosis is made accidentally, by performing a colonoscopy or enema with contrast medium.

Asymptomatic cases do not require treatment and symptomatic patients undergo dietary modifications and oral treatment. Complications require specific management, either with antibiotic or surgical treatment.

KEYWORDS: diverticulosis; mucous membrane; large intestine; colonoscopy; antibiotic.

${ }^{1}$ Médica general, graduada de la Universidad de Ciencias Médicas (UCIMED). Cód. MED13645. Correo: priella86@gmail.com

2 Médica general, graduada de la Universidad de Ciencias Médicas (UCIMED). Cód. MED12617. Correo: Kat.murilloalvarado@gmail.com

\section{INTRODUCCIÓN}

La diverticulosis colónica se describió por primera vez en 1800, era una patología casi desconocida y era observada en pacientes que se operaban por peritonitis (1).

La diverticulosis describe el desarrollo de herniaciones saculares de la mucosa y submucosa a través de áreas con capa muscular más delgada, donde los vasos sanguíneos penetran la pared intestinal (2), esta patología debe ser diferenciada de divertículos encontrados en otras áreas del tracto gastrointestinal, como los del intestino delgado, los cuales son muy infrecuentes, cerca de un $2 \%$ (3) y no se abordan en esta revisión.

Los divertículos anatómicamente se pueden clasificar en verdaderos o congénitos, cuando incluyen las tres capas de la pared colónica que son la mucosa, capa muscular y la serosa, son poco comunes (alrededor del $5 \%$ ), se ubican en el lado derecho del colon y en su mayoría ocurren en población asiática; y los divertículos falsos 0 seudodivertículos, son los más comunes (aproximadamente 95\%) y se ubican en el colon izquierdo, usualmente en el colon sigmoides (4-6).
Actualmente la diverticulosis se considera uno de los trastornos gastrointestinales más frecuentes, su prevalencia está en aumento en los países occidentales y se cree que la mitad de la población estaría afectada (7). En cuanto al cuadro clínico, alrededor de un $80 \%$ de los individuos con diverticulosis están completamente asintomáticos, el resto presenta síntomas típicos del síndrome del intestino irritable como distensión y cólicos abdominales y solo el $5 \%$ de los casos presenta complicaciones como lo son la diverticulitis y la hemorragia (8).

Usualmente el diagnostico se hace de manera accidental cuando se realiza una colonoscopia o un estudio con medio contrastado (9).

En la mayoría de los pacientes cuando son asintomáticos no se da un tratamiento específico, sin embargo, cuando presentan síntomas leves de distención y estreñimiento se recomienda aumentar la ingesta de fibra y liquido (10), pero cuando ya se presentan otros síntomas como fiebre, dolor abdominal, leucocitos; el abordaje es diferente e incluye la hospitalización, antibioticoterapia y cuando ocurren complicaciones hasta se necesitan tratamientos quirúrgicos (10). 
Esta revisión bibliografía tiene como objetivo repasar la patogenia y clínica de la diverticulosis y

hacer énfasis en el tratamiento y las complicaciones que pueden ocurrir con esta enfermedad.

\section{MÉTODO}

En esta revisión se investigó y repasó el tema de diverticulosis colónica, con sus generalidades y se escogieron bibliografías cumpliendo con los criterios de interés entre los años 2017 hasta el 2021, entre las cuales tenemos libros de texto, artículos y revistas digitales como Scielo, Elsevier, tanto en inglés como en español.

Se utilizó búsqueda entre los temas "diverticulosis colónica", "diverticulitis", "anatomía colon" "complicaciones de diverticulosis"; y con toda esta información que se obtuvo, se hizo un meticuloso análisis y se escogió lo más relevante y de mayor importancia científica para ofrecer una revisión de calidad.

\section{GENERALIDADES}

Las fibras del músculo longitudinal externo del colon conforman tres bandas largas de músculo liso conocidas como tenias colónicas. Las dos tenias anti mesentéricas (la tenia epiploica y la tenia libre) y la tenia mesentérica única, forman las hileras donde usualmente se forman los divertículos (11). Los divertículos no surgen de manera aleatoria alrededor de la circunferencia del colon, sino que se originan en cuatro hileras características que responden con los cuatro lugares de penetración de la pared intestinal muscular a la submucosa por las ramas principales de los vasos rectos, a cada lado de la tenia mesentérica y en las caras mesentéricas de las tenias epiploica y libre (11). Los divertículos mantienen esta relación anatómica fija con las tenias y es característica su ausencia de la porción del colon situada entre las dos tenias anti mesentéricas.

El uso habitual del término divertículo es incorrecto, los divertículos verdaderos contienen todas las capas de la pared intestinal. La mayoría de los divertículos del colon son, en realidad, seudodivertículos ya que están formados por herniaciones de la mucosa y submucosa a través de la capa muscular del colon por la debilidad anatómica cuando penetran los vasos sanguíneos a la pared muscular (12).

Los divertículos varían en número, desde uno hasta literalmente centenares de ellos y su diámetro habitual de un divertículo es de 3-10 mm, pero puede ser mucho mayor.

Los divertículos gigantes del colon se han definido como los que tienen un diámetro superior a $4 \mathrm{~cm}$; se han descrito tamaños de hasta $25 \mathrm{~cm}$, estos divertículos son raros; la bibliografía contiene menos de 200 casos, y se han asociado en un $30 \%$ a presentar complicaciones (11).

La geografía parece un determinante anatómico del lugar donde se encuentran los divertículos del colon. En los países occidentales se ha descrito un predominio izquierdo; aproximadamente el $90 \%$ de los enfermos presentan diverticulosis en este lado del colon, en cambio las personas de los países asiáticos tienden a presentar una diverticulosis predominantemente derecha en un $75-85 \%$ de las ocasiones.

Aunque se desconocen los factores precisos que determinan este predominio segmentario, derecho o izquierdo, en Occidente y Oriente, respectivamente, se cree que los elementos ambientales y genéticos contribuyen.

\section{ETIOLOGÍA}

La patogenia de la diverticulosis parece ser multifactorial y no se conoce bien (2). 
La explicación predominante para la formación de divertículos colónicos postula que la alteración

de la motilidad intestinal que conduce a un aumento de la presión intraluminal y esto provoca las protuberancias de la mucosa adyacente a los vasos sanguíneos (12).

Los factores ambientales que influyen en la presencia de diverticulosis, que no se habían reconocido con anterioridad y que hoy se conocen son una alimentación con poca fibra y mucha carne roja; los volúmenes fecales más bajos observados con las dietas bajas en fibra incrementaban la presión intraluminal y predisponían a la herniación diverticular (11).

Las enfermedades genéticas relacionadas con alteraciones del tejido conectivo como el síndrome de Williams Beuren, Marfan y Ehlers Danlos, estarían asociadas a diverticulosis dado cambios a nivel de la elastina19 y como esto también causa debilidad de la pared muscular (2).

\section{EPIDEMIOLOGÍA}

Es extraña antes de los 40 años (13), pero su incidencia aumenta con la edad y cerca de un $50 \%$

de los pacientes mayores de 60 años lo padecerán y es más común en el colon sigmoides (14). Es común en América del Norte, Europa y Australia, pero es poco común en Asia, África y partes de América del Sur (13).

También es más común en personas fumadora, que consumen antiinflamatorios no esteroideos y sedentarismo (12).

\section{MANIFESTACIONES CLÍNICAS}

Alrededor de un $80 \%$ de los pacientes con diverticulosis son asintomáticos a lo largo de su vida (11). Sin embargo, cuando se manifiesta la diverticulosis no complicada:

\section{Enfermedad diverticular sintomática no complicada (EDSNC)}

Usualmente cuesta establecer una relación causal entre la diverticulosis y los síntomas abdominales. Los síntomas iniciales de la EDSNC se solapan mucho con los del intestino irritable (1).

La mayoría de los enfermos con EDSNC consultan por dolor en la fosa ilíaca izquierda, el dolor suele

agudizarse con las comidas y disminuye con la deposición o la emisión de flatos. Los pacientes también refieren otros síntomas de disfunción del colon, como meteorismo, estreñimiento, diarrea o emisión de moco a través del recto. Al examen físico suele ser normal, aunque a veces se observa plenitud y ligero dolor durante la palpación de la fosa ilíaca izquierda, no se observa signo claro de rebote ni defensa (11).

Entre las complicaciones se encuentra diverticulitis, hemorragia diverticular y colitis segmentada asociada a diverticulosis abordados en complicaciones.

\section{DIAGNÓSTICO}

Como describe Vásquez Millán et al., "Importante preguntar por los antecedentes personales como hábitos dietéticos (ingesta de fibra, grasas, carne roja, etc.) y los estilos de vida (ejercicio físico) para detectar posibles factores de riesgo relacionados con la enfermedad diverticular. También es importante recoger la historia de la enfermedad, tiempo de evolución, características y localización del dolor, factores que lo alivian o empeoran y alteraciones en el hábito intestinal, entre otras" (13).

En el caso de diverticulosis sintomática a nivel del examen físico se puede encontrar, sensación de plenitud o dolor a nivel de fosa ilíaca izquierda sin datos de peritonismo. (13). 
En cuanto a los exámenes complementarios se puede realizar (13):

- Analítica: hemograma y bioquímica suelen ser normales, a menos que se presente complicaciones inflamatorias como leucocitosis, proteína $\mathrm{C}$ reactiva.

- Radiografía simple de tórax y abdomen: útil para descartar otros cuadros de abdomen agudo.

- Ecografía abdominal: para descartar otras patologías.

En base al cuadro clínico se tiene de elección:

\section{Diverticulosis no complicada}

- Endoscopía es el estudio de elección, sobre todo para descartar una neoplasia, en ocasiones, al endoscopista le puede costar navegar por un colon repleto de divertículos debido al espasmo, estenosis luminal, fijación por inflamación previa y fibrosis o confusión entre las aberturas laminares y diverticulares (11).
- Enema opaco. Sigue siendo útil en casos como cuando una colonoscopia completa no es posible o segura. En este estudio se visualizan con forma de frasco rellenado por heces o moco $(11,13)$.

\section{TRATAMIENTO}

1. Diverticulosis

a. Totalmente asintomático, usualmente no requiere tratamiento (11), sin embargo, se tiene las siguientes recomendaciones:

i. Dieta: alta es fibra es lo recomendable (15).

ii. Farmacología: no hay evidencia del uso de tratamiento farmacológico en enfermedad asintomática.

b. Tratamiento de la enfermedad diverticular sintomática se encuentra en la tabla 1.

Tabla 1. Tratamiento farmacológico de la enfermedad diverticular sintomática.

\begin{tabular}{|c|c|c|}
\hline Fármaco & Dosis & Comentario \\
\hline Rifaximina & $800 \mathrm{mg} / \mathrm{d}$ por 7 días & $\begin{array}{l}\text { Eficaz para prevenir recurrencias de la } \\
\text { enfermedad diverticular sintomática } \\
\text { no complicada y recurrencias en } \\
\text { diverticulitis. }\end{array}$ \\
\hline $\begin{array}{l}\text { Acido 5- aminosalicílico (5- } \\
\text { ASA) }\end{array}$ & $\begin{array}{l}400-800 \mathrm{mg} / \mathrm{d} \text { por } 10 \text { días } \\
\text { durante un año }\end{array}$ & $\begin{array}{l}\text { Eficaz en casos de colitis } \\
\text { ulcerativa y enfermedad de Crohn } \\
\text { en casos de inflamación grave o } \\
\text { franca. } \\
\text { Disminuye las recurrencias de la } \\
\text { enfermedad sintomática ydiverticulitis. }\end{array}$ \\
\hline Probióticos & - & $\begin{array}{l}\text { Basado en la patogenia puede } \\
\text { jugar un papel alteraciones en } \\
\text { lamicrobióta, se recomienda el uso } \\
\text { de probióticos. } \\
\text { La combinación de Lactobacilos y } \\
\text { rifaximia parece efectiva para reducir } \\
\text { las formas graves de diverticulitis y } \\
\text { prevenir lasrecurrencias. }\end{array}$ \\
\hline
\end{tabular}

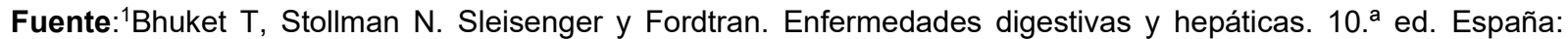

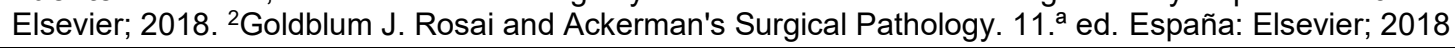




\section{COMPLICACIONES}

Entre las tres complicaciones más frecuentes se cuenta con:

\section{Diverticulitis aguda}

Se define como una inflamación y/o infección de un divertículo, este proceso se ha relacionado a que el saco diverticular se obstruye por heces espesadas en su cuello; el fecalito erosiona la mucosa del saco, produce una inflamación de bajo grado y obstruye su drenaje (11).

Aproximadamente un 10 a $25 \%$ de los pacientes la presentará y como principal factor de riesgo se encuentra el tabaco El cuadro clínico típico es dolor abdominal en el cuadrante inferior izquierdo, fiebre y leucocitos. En pacientes con divertículos en sigmoides y en asiáticos el dolor puede localizarse más en el cuadrante inferior derecho o área suprapúbica. Alteraciones en el hábito defecatorio como diarrea o constipación, así como presión pélvica puede ocurrir $(4,6,12)$.

El diagnostico se realiza con tomografía axial computarizada (TAC) con medio de contraste de abdomen es el diagnóstico de elección, con una sensibilidad de $98 \%$ y especificidad de $99 \%$, con hallazgos de engrosamiento de la pared colónica, invasión de la grasa peri colónica, abscesos, aire localizado, aire libre o líquido (12).

La clasificación se explica en la tabla 2.

El diagnóstico se utiliza TAC $O$ la endoscopía, y el tratamiento sebasa en la clasificación de Hinchey modificada, explicada en la tabla 3.

Esquema de antibióticos (13):

- Vía oral (7-10 días)

- Ciprofloxaciona 500 mg cada 12 horas y metronidazol $500 \mathrm{mg}$ cada 8 horas
- Amoxicilina más ácido clavulánico 875/125 mg cada 12 horas

- Vía intravenosa

- Clindamicina/metronidazol + quinolona

- Ampicilina-sulbactam

- Piperacilina-Tazobactam

2. Colitis segmentaria asociada la diverticulosis

- Prevalencia se encuentra entre 0,3$1.3 \%$, de los cueles, $58,7 \%$ de los enfermos son hombres y la edad media corresponde a 63.6 años (11).

- Sintomatología consiste en retortijones en la fosa ilíaca izquierda, diarrea y afectar sobre todo el colon sigmoides (11).

- En la biopsia se encuentran hallazgos de infiltración linfocítica crónica, criptitis, abscesos en las criptas e incluso granulamos (11).

- Tratamiento: 5ácido 5-aminosalicílico, en donde un $80 \%$ de los casos posee una remisión clínica (11).

\section{Hemorragia diverticular}

- Segunda causa más común de complicaciones en la enfermedad diverticular, hasta un $15 \%$ de los pacientes (6).

- No se logra localizar el divertículo responsable, y suele ser un diagnóstico de sospecha y exclusión (11).

- La hemorragia se suele producir por la erosión de la pared de una arteria en el fondo de un divertículo. La hemorragia suele ser aguda y en algunos casos masiva (11).

- Clínica: hematoquecia brusca, indolora y autolimitada, siendo el volumen de sangre moderado o grande, debido a que el vaso sangrante es una arteriola. La hemorragia diverticular es franca, una prueba positiva de sangre oculta en heces o una anemia ferropénica jamás deben atribuirse a una hemorragia diverticular. 
- Diagnóstico: se debe aclarar el diagnóstico de sospecha y localizar el foco hemorrágico, ya sea con colonoscopia, gammagrafía nuclear y angiografía.
- Tratamiento: estabilidad hemodinámica y medidas de reposición de líquidos, así como modalidad terapéutica se tiene colonoscopia, angiografía y cirugía.

\begin{tabular}{|c|c|}
\hline Complicada & No complicada \\
\hline $\begin{array}{l}\text { - Se refiere inflamación acompañada de } \\
\text { abscesos, fístulas, obstrucción o perforación } \\
\text { (ocurre en el } 5 \% \text { de los casos. } \\
\text { - Flemón/absceso, principalmente en } \\
\text { diverticulitis crónica. }\end{array}$ & $\begin{array}{l}\text { - Inflamación sin ninguna otra complicación } \\
\text { asociada. }\end{array}$ \\
\hline \multicolumn{2}{|c|}{$\begin{array}{l}\text { Fuente. }{ }^{1} \text { Reynolds J, Ward P, Rose S. Netter Collection of Medical Illustrations: Digestive System: Part II - Lower } \\
\text { Digestive Tract. 2. }{ }^{\text {a }} \text { ed. España: Elsevier ; 2017. }{ }^{2} \text { Goldblum J. Rosai and Ackerman's Surgical Pathology. } 11 .^{\mathrm{a}} \text { ed. } \\
\text { España: Elsevier; } 2018 .{ }^{3} \text { Joaquim N, Caldeira P, Antunes A. Risk factors for severity and recurrence of colonic } \\
\text { diverticular bleeding. Revista Española de Enfermedades Digestivas [Internet]. } 2017\end{array}$} \\
\hline
\end{tabular}

\begin{tabular}{|c|c|c|c|}
\hline Estadio & Definición & Hallazgos tomográficos & Tratamiento \\
\hline 0 & $\begin{array}{l}\text { Diverticulitis clínica leve } \\
\text { (diverticulitis sintomática } \\
\text { no complicada) }\end{array}$ & $\begin{array}{l}\text { Divertículos, con o sin } \\
\text { aumento asociado del } \\
\text { espesor de la pared colónica }\end{array}$ & \multirow{2}{*}{$\begin{array}{l}\text { Hemodinámica mente estable } \\
\text { y ambulatorio: antibióticos } \\
\text { intravenoso o via oral (contra } \\
\text { gram negativos yanaerobios). Si } \\
\text { empeoran síntomas y sedebe } \\
\text { realizar TAC para descartar } \\
\text { complicación. } \\
\text { Hospitalizado: antibióticos, } \\
\text { estudios, pruebas periódicas }\end{array}$} \\
\hline la & $\begin{array}{l}\text { Inflamación pericólica } \\
\text { confinada, flemón }\end{array}$ & $\begin{array}{l}\text { Aumento del espesor de la } \\
\text { pared colónica con cambios } \\
\text { en los tejidos blandos } \\
\text { pericólicos }\end{array}$ & \\
\hline Ib & $\begin{array}{l}\text { Absceso pericólico o } \\
\text { mesocólico }\end{array}$ & $\begin{array}{l}\text { Mismos hallazgos el estadio } \\
\text { la, más absceso local. }\end{array}$ & \multirow{2}{*}{$\begin{array}{l}\text { Abscesos menores de4cm: } \\
\text { antibióticos, al noresponder bien } \\
\text { al drenaje percutáneo. Si no, } \\
\text { antibióticos intravenosos. } \\
\text { Mayores de } 4 \mathrm{~cm} \text { : } \\
\text { antibióticos, drenaje.TAC } \\
\text { de seguimiento cuando el } \\
\text { drenaje es menor de } 30 \mathrm{ml} \text {. } \\
\text { Si es estado general del paciente } \\
\text { empeorasigmoidectomía electiva }\end{array}$} \\
\hline II & $\begin{array}{l}\text { Absceso pélvico, } \\
\text { intraabdominal distante } \\
\text { o retroperitoneal }\end{array}$ & $\begin{array}{l}\text { Mismos hallazgos del } \\
\text { estadio la, más absceso } \\
\text { distante anatómicamente } \\
\text { diferenciado }\end{array}$ & \\
\hline III & $\begin{array}{ll}\text { Peritonitis } & \text { purulenta } \\
\text { generalizada } & \end{array}$ & $\begin{array}{l}\text { Presencia de aire libre con } \\
\text { líquido que no se limita a una } \\
\text { cavidad abscesificada; } \\
\text { aumento del espesor de la } \\
\text { pared peritoneal }\end{array}$ & \multirow[t]{2}{*}{$\begin{array}{l}\text { Tratamiento quirúrgico de } \\
\text { emergencia sin demora. }\end{array}$} \\
\hline IV & $\begin{array}{l}\text { Peritonitis } \\
\text { generalizada }\end{array}$ & $\begin{array}{l}\text { Imposibles de distinguir de } \\
\text { los hallazgos del estadio III }\end{array}$ & \\
\hline
\end{tabular}

Fuente. ${ }^{1}$ Leeds I, Fang S. Terapias quirúrgicas actuales. 13. ${ }^{a}$ ed. España: Elsevier ; $2020 .{ }^{2}$ Young T. Diverticulitis. The New England Journal of Medicine [Internet]. 2018 [citado 18 noviembre 2020];(379) 


\section{CONCLUSIONES}

La diverticulosis es un hallazgo frecuente en población mayor de 60 años, en la mayoría de las ocasiones hallazgo incidental, ya que en un $80 \%$ de los casos es asintomático; se divide en verdaderos que posee las tres capas de la pared del colon y falsos o pseudodiverticulos con dos capas herniadas, siendo el más frecuente.

Varían en tamaño, el promedio usual 3-10 $\mathrm{mm}$ y se denomina diverticulosis gigante a un diámetro superior a $4 \mathrm{~cm}$, así como en localización mayormente izquierdo en occidente y colon izquierdo en oriente. Con etiología es variable y multi factorial. EI diagnóstico se basa en estudios endoscópicos o de imágenes médicas como TAC o estudios con medio de contraste.

Las complicaciones, siendo la más frecuente la diverticulitis cuadro típico no complicado de dolor abdominal, fiebre y leucocitosis, así como el complicado con procesos como abscesos, fístulas, obstrucción o perforación. Utilizando la clasificación de Hinchey modificada para el estadiaje y tratamiento. Seguido por la diverticular causa muy frecuente de sangrado digestivo bajo y por último, la colitis segmentarías asociada a la diverticulosis donde su sintomatología afecta con diarrea y dolor en fosa lliana derecha.

Finalmente, el tratamiento varía desde cambios en la dieta, o en sus complicaciones se adjunta antibiótico terapia e inclusive cirugía.

\section{Los autores declaran no tener conflicto de interés.}

\section{REFERENCIAS}

1. Yáñez E, Maturana J, Briones L. Enfermedad diverticular: nuevas perspectivas en el tratamiento dieto-terapéutico. Revista chilena de nutrición [Internet]. 2019 [citado 18 noviembre 2020];(46). Disponible en: https://www.scielo.cl/scielo.php?script=sci arttext \&pid=S0717-75182019000500585\&lang=es
2. Fluxá D, Quera R. Enfermedad diverticular: mitos y realidades. Revista médica de Chile [Internet]. 2017 [citado 18 noviembre 2020];(145). Disponible en: https://www.scielo.cl/scielo.php?script=sci arttext \&pid=S0034-98872017000200009\&lang=es

3. Valdés M, Gutierrez A, Gil M. Dolor abdominal en paciente con antecedente de diverticulosis colónica. Sanidad Militar [Internet]. 2019 [citado 18 noviembre 2020];(75). Disponible en: https://scielo.isciii.es/scielo.php?script=sci arttext \&pid=S1887-85712019000300172\&lang=es

4. Roccatagliata N, Rodríguez L, Guardo L. Revisión de la clasificación de Hinchey (diverticulitis) y su correlación terapéutica. Revista argentina de radiología [Internet]. 2020 [citado 18 noviembre 2020];(84). Disponible en: http://www.scielo.org.ar/scielo.php?script=sci artt ext\&pid=S1852-99922020000400123\&lang=es

5. Raña-Garibay R, Salgado-Nesme N, CarmonaSánchez R, Remes-Troche J. Consenso mexicano sobre el diagnóstico y tratamiento de la enfermedad diverticular del colon. Revista de Gastroenterología de México. 2019;(84). Disponible en: https://www-clinicalkeyes.binasss.idm.oclc.org/\#!/content/journal/1-s2.0S037509061930045X

6. Shields H, Scheid F, Pierce T, Andersson K. Case 4-2019: An 18-Year-Old Man with Abdominal Pain and Hematochezia. The New England Journal of Medicine [Internet]. 2019 [citado 18 noviembre 2020];(380). Disponible en: https://www-nejmorg.binasss.idm.oclc.org/doi/full/10.1056/NEJMcp c1810391

7. Didnée A, Meurette G. Técnicas quirúrgicas Aparato digestivo. TRATADOS EMC [Internet]. 2020 [citado 18 noviembre 2020];(36). Disponible en: https://www-clinicalkeyes.binasss.idm.oclc.org/\#!/content/emc/51-s2.0S128291292043990X

8. Rozman C, Cardellach F. Medicina Interna. 19. ${ }^{a}$ ed. España: Elsevier; 2020. Disponible en: https://www-clinicalkeyes.binasss.idm.oclc.org/\#!/content/book/3-s2.0B9788491135456000193?scrollTo=\%23hl000028 2

9. Reynolds J, Ward P, Rose S. Netter Collection of Medical Illustrations: Digestive System: Part II Lower Digestive Tract. 2. ${ }^{a}$ ed. España: Elsevier ; 2017. Disponible en: https://www-clinicalkeyes.binasss.idm.oclc.org/\#!/content/book/3-s2.0B9781455773916000032?scrollTo=\%23hl000049 $\underline{0}$

10. Leeds I, Fang S. Terapias quirúrgicas actuales 13. ${ }^{a}$ ed. España: Elsevier ; 2020. Disponible en: https://www-clinicalkeyes.binasss.idm.oclc.org/\#!/content/book/3-s2.0- 
B9788491137443000313?scrollTo=\%23hl000013 1

11. Bhuket T, Stollman N. Sleisenger y Fordtran. Enfermedades digestivas y hepáticas. 10. ${ }^{\mathrm{a}}$ ed. España: Elsevier; 2018. Disponible en: https://www-clinicalkey-

es.binasss.idm.oclc.org/\#!/content/book/3-s2.0B9788491132110001212?scrollTo=\%23hl000022 $\underline{9}$

12. Young T. Diverticulitis. The New England Journal of Medicine [Internet]. 2018 [citado 18 noviembre 2020];(379). Disponible en: https://www-nejmorg.binasss.idm.oclc.org/doi/full/10.1056/NEJMcp 1800468

13. Goldblum J. Rosai and Ackerman's Surgical Pathology. 11. a ed. España: Elsevier; 2018. Disponible en: https://www-clinicalkeyes.binasss.idm.oclc.org/\#!/content/book/3-s2.0B9780323263399000172?scrollTo=\%23hl000125 $\underline{2}$

14. Reynolds J, Ward P, Rose S. Netter Collection of Medical Illustrations: Digestive System: Part II Lower Digestive Tract. 2. ${ }^{a}$ ed. España: Elsevier ; 2017. Disponible en: https://www-clinicalkeyes.binasss.idm.oclc.org/\#!/content/book/3-s2.0B9781455773916000032?scrollTo=\%23hl000049 $\underline{0}$

15. Schlienger J. Dietética en la práctica médica. 2 . $^{\text {a }}$ ed. España: Elsevier; 2018. Disponible en: https://www-clinicalkeyes.binasss.idm.oclc.org/\#!/content/book/3-s2.0B9788491132714000227

16. Joaquim N, Caldeira P, Antunes A. Risk factors for severity and recurrence of colonic diverticular bleeding. Revista Española de Enfermedades Digestivas [Internet]. 2017 [citado 18 noviembre 2020];(109). Disponible en: https://scielo.isciii.es/scielo.php?script=sci arttext \&pid=S1130-01082017000100002\&lang=es 